\title{
COMPORTAMENTO DO HERB ICIDA METRIBUZIN, APLICADO ISOLADO E EM MISTURA COM OUTROS HERBICIDAS, NO \\ CONTROLE DE PLANT AS DANINHAS EM CAFEEIROS NOVOS \\ POR DOIS ANOS CONSECUTIVOS
}

\author{
L.S.P. CRUZ ${ }^{1} \&$ C.A.L. SANTOS ${ }^{2}$ \\ 1 Pesquisador Científico do Instituto \\ Agronômico. C.Postal 28. CEP 13.100. \\ Campinas, SP. \\ ${ }^{2}$ Pesquisad or Científico do Instituto Bioló gico. \\ C.Postal, 70 - 13.100 - Campinas, SP.
}

\section{RESUMO}

Para se conhecer o comportamento do herhicida metribuzin, aplica do isolado ou em mistura com outros herbicidas residuais (napropamide, pendimethalin, alachlor, oryzalin e diuron) no controle de plantas daninhas que comumente infestam cafeeiros em formação, foi conduzido um experimento de campo onde esse herbicida a 0,$28 ; 0,42 ; 0,56$ e $0,70 \mathrm{~kg} / \mathrm{h}$ a foi aplica do isolado e a $0,28 \mathrm{~kg} / \mathrm{ha}$ em mistura com napropamide a 4,00 kg / ha, pendime thal in a 1,00 kg/ha, alachlor a 2,40 kg/ha, oryzalin a $1,50 \mathrm{~kg} / \mathrm{ha}$ ou com diuron a 1,20 $\mathrm{kg} / \mathrm{ha}$, comparado com duas testemunhas, onde, em uma, o mato foi mantido a níveis não competitivos com a cultura, por meios mecânicos, e outra onde o mato foi deix ado desenvolver-se naturalmente, sem nenhu ma interferência. Foi incluído também um tratamento com diuron a $1,20 \mathrm{~kg} / \mathrm{ha}$.
A infestação natural de plantas daninhas da área do experimento era formada pelas gramínas, capim-de-colchão (Digitaria sanguina (L.) Scop.), capim marmelada (Bachiaria plantaginea (Link.) Hitch) capim- pé-de-galinha (Eleusine indica (L.) Gaertn.), capim-favorito (Rhynchelitrum roseum (Nees) Stapf et Hubb), e pelas dicotiledôneas beldroega (Portulaca oleracea L.), picão-branco (Galinsoga parviflora Cav.), carurú -de mancha (Amarantus virid is L.), amendoimbravo (Euphorbia hetentophylla L.), picãopreto (Bidens pilosa L.), mentruz (Lepidium virginicum L.), quebra-pedra (Phyllantus cordovadem sis Muell Arg.) e fals a-serra lha (Emilia sanchifolia DC) .

Os resultados dos dois anos foram semelhantes para cada trata mento. Metribuzin a $0,28 \mathrm{~kg} / \mathrm{ha}$ em mistura com napropamide a $4,00 \mathrm{~kg} / \mathrm{ha}$, ou com alachlor a 2,40 $\mathrm{kg} / \mathrm{ha}$, apresentaram períodos de ação maior, seguidos da mistura de me- 
tribuzin, naquela mesma dose, com oryzalin a $1,50 \mathrm{~kg} / \mathrm{ha}$.

Não foi constatado a presença de qualquer sintoma de intoxicação nos cafeeiros, em todos os tratamentos, até a última observação, realizad a 210 dias após a segunda aplicação dos herb icidas.

PALAVRAS-CHAVE: metribuzin, misturas de herbicidas, cafeeiros novos.

\section{SUMMARY}

EFFECT OF METRIBUZIN, SPRAYED ALLONEOR INMIXTUREWITHANOTHER HERBICIDES,FOR WEED CONTROLON YOUNG COFFEETREES, DURING TWO YEARS

This paper reports on two years field trials carried out in Araras, SP, Brasil, on coffee trees with twenty months of age, on clay soil.

The herbicides studied were metrihuzin at $0.28 ; 0.42 ; 0.56 ;$ and $0,70 \mathrm{~kg} / \mathrm{ha}$; metribuzin plus napropamide at $0.28+4.00$ $\mathrm{kg} / \mathrm{ha}$; metribuzin plus pendimethalin at 0.28 $+1.00 \mathrm{~kg} / \mathrm{ha}$; metribuzin plus alachlor at 0.28 $+2.40 \mathrm{~kg} / \mathrm{ha}$; metribuzin plus oryzalin at $0.28+1.50 \mathrm{~kg} / \mathrm{ha}$ and metribuzin plus diuron at $0.28+0.80 \mathrm{~kg} / \mathrm{ha}$. The applications were done in preemergen ce in March/79 and February/80.

The weeds were represented by Digitaria sanguinalis, Brachiaria plantaginea, Eleusine indica, Rhynchelitrum roseum, Portulaca oleracea, Galinsoga parviflora, Amaranthus viridis, Euphorbia heterophylla, Bidens pilosa, Lepidium virginicum, Phullanthus corcovadensis and Emilia sonchifolia.

The treatments that presented the longest action after 210 days of herbicides application was metribuzin plus napropamide, followed by metribuzin plus oryzalin.

No injury was observed by the herbicides on the coffee trees. KEYWORDS: metribuzin, herbicide mixtures, young coffee trees.

\section{INTRODUÇÃO}

A aplicação de herbicidas em programa de controle de plantas daninhas em cafeeiros é uma pratica cultural que vem se tornando cada vez mais usada no Brasil, principalmente em decorrência das dificuldades que o emprego da mão-deobra com capinas vem causando nestes últimos anos $(3,13,14)$.

A cultura do café, assim como a grande maioria das culturas, é infestada por monocotiledôneas e dicotiledôneas, sendo necessário o uso de dois ou mais herbicidas, em mistura ou combinados, para seu controle, uma vez que não é encontrado no mercado um composto de aplicação em pré-emergência que controle das plantas daninhas daquelas duas clas ses de vegetais ao mesmo tempo, e que não seja prejudicial ao desenvolvimento ou à produção dos cafe eiros .

Alguns herbicidas residuais já foram testados anteriormente para uso em cafeeiros novos sem causar prejuízos aos mesmos. Assim é que pendimethalin foi pesquisado por Fujiwara e Scholle (3) em doses variáveis de 0,10 a $2,00 \mathrm{~kg} / \mathrm{ha}$, sem causar fitotoxicidade aos cafeei ros novos. Fujiwara (4) aplic ou pendimethalin nas doses de 0,66; 0,99 e 1,32 kg/ha em cafeeiros com seis meses de idade, plantados em solo podzolisado de Lins e Marília, com pulverizador PHD-Jacto e não encontrou sintomas de intoxi- 
cação nos cafeeiros em todos os tratamentos do experimento.

Pompeu et al. (11) pesquisaram o comportamento do alachlor a 1,92; 2,40; 2,88 e $3,36 \mathrm{~kg} / \mathrm{ha}$, pendimethalin a 1,55 $\mathrm{kg} / \mathrm{ha}$, oryzalin a 1,50 e $1,88 \mathrm{~kg} / \mathrm{ha}$ e napropamide a 2,00 kg/ha, além do oxifluorfen a $1,00 \mathrm{~kg} / \mathrm{ha}$, em cafeeiros comcinco, seis e 10 meses de campo, e não encontraram sintomas de intoxicação nos mesmos. Os cafeeiros encontravam-se vigorosos, tanto em altura como em enfolhamento, após um ano de observação.

Cruz e Novo (2) empregando napropamide a 2,00 e $3,00 \mathrm{~kg} / \mathrm{ha}$ e simazine a 0,50 e $0,75 \mathrm{~kg} / \mathrm{ha}$, e suas respectivas misturas, em cafeeiros com 24 meses, encontraram sintoma de intoxicação somente para os tratamentos com simazine. Napropamide não foi prejudicial ao desenvolvimen to vegetativo dos cafeeiros.

Diuron a 1,6 kg/ha, isolado, e em mistura com oryzalin, em diversas doses, não apres entou fitotoxicidade para cafeeiros novos ou em produção, quand o aplic ado em pré-emergência dirigida, em experimentos cond uzidos por Honda et al. (8).

No Brasil, metribuzin tem sido pouco estudado em cafeeiros novos (8).

Procurou-se conhecer a ação do metrihuzin, aplicado isola do ou em mistura com outros herbicidas residuais, comprovadamente seletivos para cafeeiros novos, durante dois anos consecutivos.

\section{MATE RIAL E MÉTODOS}

$\mathrm{O}$ experimento foi conduzido em area de solo com textura argilosa, da Fazenda Pinhalzinho, Araras,SP, em cultura de café cv. Catuaí, nos anos de 1979/80.
As parcelas experimentais eram formadas por uma fileira com quatro covas de três cafeeiros cada, com área de $36,00 \mathrm{~m}^{2}$, distribuídas em um esquema de blocos casualizados, com 13 tratamentos e quatro repetições.

Metribuzin foi aplicado isolado a 0,28; 0,$42 ; 0,56$ e $0,70 \mathrm{~kg} / \mathrm{ha}$, e aplicado a 0,28 $\mathrm{kg} / \mathrm{ha}$ em mistura com napropamide a 4,00 $\mathrm{kg} / \mathrm{ha}$; pendimethalin a $1,00 \mathrm{~kg} / \mathrm{ha}$; alachlor a $2,40 \mathrm{~kg} / \mathrm{ha}$; oryzalin a $1,50 \mathrm{~kg} / \mathrm{ha}$ ou diuron a $0,80 \mathrm{~kg} / \mathrm{ha}$. Constou do experimento também um tratamento com diuton a $1,20 \mathrm{~kg} / \mathrm{ha}$. Foram incluídas duas testemunhas, uma com os cafeeiros mantidos sem a concorrência das plantas daninhas por meios mecânicos e outra onde o mato foi deixado desenvolver-se naturalmente, sem nenhuma interferência portanto.

As aplicações, em pré-emergência dirigida, foram realizadas no final do verão, em 01.03.79 em 08. 02.80, com pulverizador costal, manual, dotado de bico de jato plano da série 80.03, com gasto de calda correspondente a 300 1/ha. A primeira aplicação dos herbicidas foi feita quando as plantas de café estavam com 20 meses de idade.

A infestação natural de plantas daninhas do experimento, com $100 \%$ de frequência, era representada pelas monocotiledôneas: capimde-colchão (Digitaria sanguinalis (L.) Scop) , capim marmelada (Brachiaria plantaginea (Link) Hitch), capim-pé-de-galinha (Eleusine indica (L.) Gaertn), e capim-favorito (Rhynchelitrum roseum (Nees.) Stapf et Hubb); e, pelas dicotiledôneas: beldroega (Portulaca oleracea L.), picão-branco (Galinsoga parviflora Cav.), caruru-demancha (Amaranthus viridis L.), amendoim bravo (Euphorbia heterophylla L), 
picão-pre to Bidens pilosa L.) , mentruz (Le pidium virginicum L.) , quebra-ped ra (Phyll anthus Corcovadesis (Muell.) Arg.) e fal sa serralha (Emilia sonchifolia DC.) .

Para a avaliação da efi ciência dos herbicidas experimentados, foi considerada a porcentagem de inf estação do mato; e, para a constatação da sel etividade, for am ava liados os sin tomas de intoxicação, ambos, aos 15, 30, 45, $60,75,80,105,120,135,150$ e 210 dias após a aplicação dos herbicidas (DAT). Aos 45 dias, também foi feita a contagem de plantas daninhas por espécie botânica, em duas areas representativas da inf estação média, correspondente a $5 \%$ da área das parcelas sem he rbicida,segundo o pre conizado por Igue et al (9) .

Quanto pelo menos uma parcela do tratamento atingia $25 \%$ de infestação geral, todo o tratamento era capinado com enxada. Aos 210 dias da aplicação dos herbicidas foi feita uma última avaliação de infestação de plantas daninhas, sendo então realizada uma capina geral em todo o experimento.

\section{RESULTADOS E DISCUSSÃO}

Os efeitos dos herbicidas sobre as plantas daninhas que incidiram no experimento, mensurados pela sua porcentagem de infestação, são demonstrados pelos dados dos Quadros 1 e 2 , respectivamente para os experimentos de 1979 e 1980.

A densidade populacional das plantas daninhas, e sua correspondente infestação porcentual, após 45 dias da aplicação dos compostos foi a seguinte:

\begin{tabular}{lrrrrr} 
Espëcie botânica & $\begin{array}{c}\text { Densidade } \\
\text { Populacional } \\
(\mathrm{no/m})\end{array}$ & & \multicolumn{2}{c}{$\begin{array}{c}\text { Infestação } \\
\text { (\%) }\end{array}$} \\
\cline { 2 - 3 } \cline { 5 - 6 } & 1979 & 1980 & & 1979 & 1980 \\
\hline Portulaca oleracea & 291 & 377 & & 50,50 & 26,1 \\
Galinsoga parviflora & 88 & 153 & & 15,3 & 10,6 \\
Amaranthus viridis & 55 & 239 & & 9,5 & 16,6 \\
Euphorbia heterophylla & 47 & 27 & & 8,1 & 1,9 \\
Bidens pilosa & 29 & 348 & & 5,0 & 24,1 \\
Lepidim virginicum & 25 & 14 & & 4,4 & 1,0 \\
Phyllanthus corcovadensis & 12 & 53 & & 2,1 & 3,7 \\
Rhynchelitrum roseum & 9 & 94 & & 1,6 & 6,5 \\
Brachiaria plantaginea & 7 & 8 & & 1,2 & 0,5 \\
Emilia sonchifilia & 5 & 12 & & 0,9 & 0,8 \\
Digitaria sanguinalis & 4 & 75 & & 0,7 & 5,2 \\
Eleusina indica & 4 & 43 & & 0,4 & 3,0 \\
\hline
\end{tabular}


Quadro 1. Médias das porcentagens de infestação de planta daninha em experimento com misturas de herbicidas aplicadas em pré-emergência em cafeeiros novos. Araras, SP, 1979.

\begin{tabular}{|c|c|c|c|c|c|c|c|c|c|c|c|}
\hline \multirow{3}{*}{ Berblcida: } & \multirow{3}{*}{\multicolumn{2}{|c|}{$\begin{array}{l}\text { Dose } \\
(\mathrm{Kg} / \mathrm{ha})\end{array}$}} & \multicolumn{9}{|c|}{ Porcentagem méd1a de 1nfestação de mato } \\
\hline & & & \multicolumn{2}{|c|}{ D1 as após } & \multicolumn{2}{|c|}{ a aplicaçäo } & \multicolumn{2}{|c|}{ dos herbicidas } & \multirow[b]{2}{*}{105} & \multirow[b]{2}{*}{120} & \multirow[b]{2}{*}{210} \\
\hline & & & 15 & 30 & 45 & 60 & 75 & 90 & & & \\
\hline \multirow[t]{4}{*}{ Natribusi n } & & 0,28 & 4,00 & 4,25 & 7,75 & 13,50 & $22,25 * \bullet$ & & & & $45,00 *$ \\
\hline & & 0,42 & 2,75 & 2,75 & 6,25 & 9,50 & 13,00 & $18,75^{*}$ & & & $50,75^{*}$ \\
\hline & & 0,56 & 2,25 & 3,25 & 5,00 & 7,50 & 10,25 & $23,25 *$ & & & $60,00^{\star}$ \\
\hline & & 0,70 & 2,25 & 3,25 & 4,00 & 5,00 & 7,00 & 14,00 & $16,25^{*}$ & & $47,50^{\circ}$ \\
\hline Metribuzin + napropanlde & 0,28 & $+4,00$ & 3,00 & 3,50 & 3,75 & 5,25 & 6.50 & 7,75 & 10,00 & $12,00^{*}$ & $26,25 *$ \\
\hline Netribuxin + pendimethalln & 0,28 & $+1,00$ & 2,75 & 4,00 & 5,50 & 5,25 & 13,75 & $16,25^{*}$ & & & $38,75 *$ \\
\hline Metribuzin + alachlor & 0.28 & $+2,40$ & 1,75 & 2,00 & 3,75 & 6,50 & 8,25 & 11,00 & 11,25 & $15,00^{*}$ & $49,00 *$ \\
\hline Metribuzin + oryzalin & 0,28 & $+1,50$ & 2,25 & 3,25 & 4,00 & 5,50 & 5,50 & 9,00 & 11,00 & $14,25 *$ & $25,00^{*}$ \\
\hline metribuzin + diuron & 0.28 & $+0,80$ & 2,25 & 2,25 & 4,25 & 7,00 & 10,00 & 14,25 & $16,75^{*}$ & & $40,00 *$ \\
\hline diuron & & 1,20 & 4,50 & 6,50 & 12,50 & $17,50^{*}$ & & & & & $60,00^{*}$ \\
\hline \multirow[t]{2}{*}{ Testemunha capinada } & & & 12,00 & 13,25 & 22,50 & $41,25^{\star}$ & & & & & $60,00 *$ \\
\hline & & & 11,00 & 16,00 & 20,75 & $32,50^{*}$ & & & & & $66,25 *$ \\
\hline Testemunha sem cap1 & & & 12,00 & 12,25 & 21,50 & 33,75 & 60,00 & 45,00 & 45,00 & 47,25 & 65,00 \\
\hline
\end{tabular}

- Capinado com enxada nesta data. 
Quadro 2. Médias das porcentagens de infestação de plantas daninhas em experimento com misturas de herbicidas de aplicação em pré-emergência, em cafeeiros novos. Araras, SP, 1980.

\begin{tabular}{|c|c|c|c|c|c|c|c|c|c|c|c|c|}
\hline \multirow{3}{*}{ Berb1c1das } & \multirow{3}{*}{$\begin{array}{l}\text { Doses } \\
(\mathrm{kg} / \mathrm{ha})\end{array}$} & \multicolumn{11}{|c|}{ 'Porcentagem média de infestacão do mato } \\
\hline & & \multicolumn{11}{|c|}{ 'D1as após a aplicação dos herb1c1das } \\
\hline & & 15 & 30 & 45 & 60 & 75 & 90 & 105 & 120 & 135 & 150 & 210 \\
\hline \multirow[t]{4}{*}{ Metribuzin } & 0,28 & 15,25 & 20,50 & 21,00 & 22,00 & $25,50 *$ & & & & & & $38,50^{*}$ \\
\hline & 0,42 & 8,50 & 13,75 & 14,25 & 15,00 & 15,00 & 20,00 & $22,50 *$ & & & & 30,25 \\
\hline & 0,56 & 8,75 & 13,25 & 13,75 & 15,00 & 15,75 & 19,25 & 22,75 * & & & & $3 c, 50^{*}$ \\
\hline & 0,70 & 8,00 & 13,00 & 14,50 & 16,00 & 16,25 & 18,75 & 20,00 & $22,00^{*}$ & & & $32,00^{*}$ \\
\hline mtribuzin + napropamide & $0,28+4,00$ & 5,25 & 7,75 & 8,00 & 8,25 & 9,00 & 9,25 & 15,00 & 17,00 & 19,25 & $22,75^{*}$ & $15,00^{*}$ \\
\hline Metribuzin + pendimethallen & $0,28+1,00$ & 6.00 & 8,75 & 10,25 & 12,50 & 15,00 & 17,50 & 19,00 & $21,75^{*}$ & & & $18,50^{*}$ \\
\hline Metribuzin + alachlor & $0,28+2,40$ & 4,75 & 6,25 & 7,75 & 8,00 & 15,00 & 17,50 & 18,25 & 19,25 & 20,75 & $25,00 *$ & 18,50 \\
\hline Metribuzin + oryzalin & $0,28+1,50$ & 5,25 & 7,50 & 8,50 & 10,00 & 12,25 & 17,00 & 17,75 & 20,50 & $22,75 *$ & & 25,25 * \\
\hline Metribuzin + diuron & $0,28+0,80$ & 12,25 & 20,25 & 20,50 & 20,75 & 21,50 & $22,75 *$ & & & & & $40,00^{*}$ \\
\hline Di uron & 1,20 & 17,25 & $25,20^{*}$ & & & & & & & & & $58,75^{*}$ \\
\hline \multirow[t]{2}{*}{ Testemunha capinada } & & 18,50 & $25,00 *$ & & & & & & & & & $62,00^{*}$ \\
\hline & & 21,75 & $24,50^{*}$ & & & & & & & & & 51,25 \\
\hline Testemunha sem capina & & 22,75 & 41,75 & 43,25 & 45,00 & 52,00 & 55,75 & 60,00 & 65,50 & 65,75 & 65,00 & 75,00 \\
\hline
\end{tabular}

- Capinadu com enxada nesta data. 
A análise da porcentagem de infe stação do mato (Quadros 1 e 2) indica que os resultados dos dois anos foram semelhantes para cada tratamento, sendo que no primeiro ano, quando a aplic ação dos herbicidas foi realizada mais próxima do período seco do ano, seus efeitos sobre as espécies daninhas foram mais prolongados.

Aos 60 DAT, no primeiro ano, e 30, no segundo, o tratamento com diuron apresentava parcela com $25 \%$ de infe stação, necessitando de limpeza, a qual coincidiu com a necessidade de capina das testemunhas. Resultados de pesquisas anteriores $(1,5,12)$ indicaram um período de ação maior para o diuron, se bem que bastante variável. Assim que Sheets \& Harris (12) citam resulta dos de campo de Hollings worth (7), Weld on \& Tommons $\mathrm{e}$

Nearures

(10) dando como de cinco a sete meses a fitotoxicidade residual do diuron, usado de 3,6 a 4,0 kg/ha. Hill et al. (6), empre gando doses mais baixas, de 1,0 a $2,0 \mathrm{~kg} / \mathrm{ha}$ de diuron, encontraram resíduo variando de quatro a oito meses. Weldon \& Timmons (15) trabalhando com apenas $2,00 \mathrm{~kg} / \mathrm{ha}$ de diuron, encontraram resíduo desse herbicida ata 15 meses após a aplic ação. Esses dados de resíduo podem ser comparados com a duração do efeito do diuron.

A causa do baixo período de controle do diuron no experimento, de um a dois meses, não foi determinada, podendo-se atribuir o fato à baix a dose empre gada para solo argiloso, ou às condições climáticas ocorridas por ocasião da aplicação.

Quando diuron foi aplicado em mistura com metribuzin teve sua ação de controle melhorada. Metribuzin, na mesma dose utilizada namistura com diuron, quando usado isolado, também ofereceu resultados inferiores.

Os dados dos Quadros 1 e 2 indicam que os melhores resultados de controle geral das plantas daninhas foram obtidos com a mistura de metribuzin a $0,28 \mathrm{~kg} / \mathrm{ha}$ com napropamide a 4,00 kg/ha. Cruz et al. (1) e Cruz e Novo (2), encontraram bons resultados de controle de plantas daninhas empregando napropamide a 2,00 e 3,00 kg/ha. Neste experimento, napropamide foi empregado em dose mais elevada na mistura para se obter uma persistência mais prolongada e conhecer os efeitos dessa dose sobre os cafeeiros.

As misturas de metribuzin a $0,28 \mathrm{~kg} / \mathrm{ha}$ com alachlor a $2,40 \mathrm{~kg} / \mathrm{ha}$ e com oryzalin a $1,50 \mathrm{~kg} / \mathrm{ha}$ apresentaram resultados pouco inferiores ao do metribuzin a $0,28 \mathrm{~kg} / \mathrm{ha}+$ napropamide a $4,00 \mathrm{~kg} / \mathrm{ha}$.

A ação de controle das plantas daninhas oferecidas por metribuzin a $0,28 \mathrm{~kg} / \mathrm{ha}+$ pendimethalin a $1,00 \mathrm{~kg} / \mathrm{ha}$ foi maior do que a de metribuzin a $0,28 \mathrm{~kg} / \mathrm{ha}+$ diuron a 0,80 $\mathrm{kg} / \mathrm{ha}$ e inferior à das demais misturas, assim como de metribuzin a $0,70 \mathrm{~kg} / \mathrm{ha}$, usado isolado.

Não foi observado qualquer efeito fitotóxico dos herbicidas sobre as plantas de café, até a última avaliação, realizada 210 dias após asegunda aplicação dos herbicidas.

Os resultados obtidos permitem concluir que: 1) as misturas de metribuzin a $0,28 \mathrm{~kg} / \mathrm{ha}$ com napropamide a 4,00 kg/ha, ou com alachlor a $2,40 \mathrm{~kg} / \mathrm{ha}$, ou ainda com oryzalin a $1,50 \mathrm{~kg} /$ há apresentaram os maiores períodos de controle do mato; 2) a persistência de metribuzin a $0,28 \mathrm{~kg} / \mathrm{ha}$, empregado em mistura com pendimethalin a $1,00 \mathrm{~kg} / \mathrm{ha}$ para o controle das espécies infestantes foi inferior ao das misturas anteriormente citadas; 3) metribuzin a $0,28 \mathrm{~kg} / \mathrm{ha}+$ 
diuron a $0,80 \mathrm{~kg} / \mathrm{ha}$ apresentou o menor período de controle quando comparado com as misturas de metribuzin e napropamide, ou alachlor, ou oryzalin, ou pendimethalin, e 4) as misturas, assim como o metribuzin e diuron, empre gados isolados, não prejudicaram o desenvolvimento vegetativo dos cafeeiros novos durante os dois anos de duração do experimento.

\section{LITERATURA CITADA}

01. Cruz, L.S.P.; Leiderman, L. \& Molinari, J.A.B. Primeiros resultados de controle de plantas daninhas com o herbicida napropamide em cafeeiros. Série Experimentação Cafeeira, 1(3): 19$29,1977$.

02. Cruz, L.S.P. \& Novo, M.C.S.S. Efeitos de misturas de napropamide e simazine no controle de mono e dicotile dôneas em cafeeiros novos. In: Congr. Bras. Herb. Ervas Dan., 139, Ilhéus, Itabuna, BA, Resumos, p.29-30. 1980 .

03. Fujiwara, M. \& Sholle, R. K. Nota sobre o uso de penoxa lin na formação de culturā de café. In: Sem.Bras.Herb.

Ervas Dan., 119, Londrina. Resumos, p.59. 1976.

04. Fujiwara, M. Nota sobre a aplicação do penoxalin com - pulverizador PHD-Jacto em cafeeiro em formação. In:

Congr. Bras. Pesq. Caf., 40 Caxambú, MG, Resumos, p. 100. 1976.

05. Hertwig, K. von. Manual de Herbicidas, Desfolhantes, dessecantes e fitoreguladores, Editora Agronômica Ce res Ltda., lạ ediçäo, 1977. 480pp.

06. Hil1, G.D.; McGahem, J.W.; Baker, H.M.; Finnerty, D. W. \& Bingeman, C.W. The fate of substituted urea herbici des in agricultural soils. Agron. J., 47: 93, 1955.

07. Hollingsworth, E.B. Cottan res ponse to two substituted u= reas and CIPC and their per sistence and movement in soil. In: Proc. S. Weed Conf., 8a., p.294. 1955.

08. Honda, T.; Pompeu, R.M. \& 01iveira, I.A.M. Efeito do herbicida oryzalin no controle das ervas daninhas do cafeeiro (Coffea arabica L). In: Sem. Bras. Herb. Ervas Dan., 11ㅇ, Londrina, PR. Re sumos, pp. 57-58, 1976.

09. Igue, T., Forster, R. \& Deuber, R. Amostragem mínima em contagem de ervas em experimentos com herbicidas. In: Sem. Bras. Herb. Ervas Dan., 109, Santamaria, RS. Resumos, p.54. 1974.

10. Neururer, H. Nachwirkung der Vorjahringen Simazin und Gesaprim-Sprintzung zu mais . Pflanzenart, 14: 76. 1962.

11. Pompeu, R.M.; Lusvarghi, H.; Kashiwakura, Y. \& Ferré, J.L.E. Efeitos da aplica ção de alachlor e outros her bicidas em cafeeiros novos.

In: Congr. Bras. Herb. Ervas Dan., 13\%, I1héus/It abu na, BA. Resumos, pp.26-27. 1980 .

12. Sheets, T.J. \& Harris, C.I.Her bicides, residues in soils and their phytotoxicities to crops grown in rotations. Res. Rev., 11: 119-140. 1965.

13. Silveira, G.M.; Kurachi, S.A. 
H.; Moraes, M.V. \& Grohmam, F. Métodos mecânicos e quí micos de controle de ervas daninhas em cafezal. In: Congr. Bras. Pesq. Caf., 2\%, Poços de Caldas, MG, Resumos, pp.337-339. 1974.
14. Ueda, A. Anālise do comporta mento das triazinas a diferentes niveis na cafeicultu ra. In: Sem. Bras. Herb. E $\bar{r}$ vas Dan., $11 \%$, Londrina. Rē sumos, pp. $60-61$. 1976. 